\title{
Physical and chemical nature of the scaling relations between adsorption energies of atoms on metal surfaces
}

\author{
Calle-Vallejo, F.; Martínez, J. I.; García Lastra, Juan Maria; Rossmeisl, J.; Koper, M. T. M.
}

Published in:

Physical Review Letters

Link to article, DOI:

10.1103/PhysRevLett.108.116103

Publication date:

2012

Document Version

Publisher's PDF, also known as Version of record

Link back to DTU Orbit

Citation (APA):

Calle-Vallejo, F., Martínez, J. I., García Lastra, J. M., Rossmeisl, J., \& Koper, M. T. M. (2012). Physical and chemical nature of the scaling relations between adsorption energies of atoms on metal surfaces. Physical Review Letters, 108(11), 116103. https://doi.org/10.1103/PhysRevLett.108.116103

\section{General rights}

Copyright and moral rights for the publications made accessible in the public portal are retained by the authors and/or other copyright owners and it is a condition of accessing publications that users recognise and abide by the legal requirements associated with these rights.

- Users may download and print one copy of any publication from the public portal for the purpose of private study or research.

- You may not further distribute the material or use it for any profit-making activity or commercial gain

- You may freely distribute the URL identifying the publication in the public portal 


\title{
Physical and Chemical Nature of the Scaling Relations between Adsorption Energies of Atoms on Metal Surfaces
}

\author{
F. Calle-Vallejo, ${ }^{1}$ J. I. Martínez, ${ }^{2,3}$ J. M. García-Lastra, ${ }^{3}{ }$ J. Rossmeisl, ${ }^{3}$ and M. T. M. Koper ${ }^{1, *}$ \\ ${ }^{1}$ Leiden Institute of Chemistry, Leiden University, P.O. Box 9502, 2300 RA Leiden, The Netherlands \\ ${ }^{2}$ Departamento de Física Teórica de la Materia Condensada, UAM, ES-28049 Madrid, Spain \\ ${ }^{3}$ Center for Atomic-scale Materials Design, Department of Physics, DTU, DK-2800 Kongens Lyngby, Denmark
}

(Received 12 September 2011; published 16 March 2012)

\begin{abstract}
Despite their importance in physics and chemistry, the origin and extent of the scaling relations between the energetics of adsorbed species on surfaces remain elusive. We demonstrate here that scalability is not exclusive to adsorbed atoms and their hydrogenated species but rather a general phenomenon between any set of adsorbates bound similarly to the surface. On the example of the near-surface alloys of Pt, we show that scalability is a result of identical variations of adsorption energies with respect to the valence configuration of both the surface components and the adsorbates.
\end{abstract}

DOI: 10.1103/PhysRevLett.108.116103

PACS numbers: 82.65.+r, 82.37. $-\mathrm{j}, 82.60 .-\mathrm{s}$

The discovery of linear relations between the adsorption energies of atoms and their hydrogenated species on transition metal surfaces is one of the major advances of theoretical surface science and heterogeneous catalysis in the past decade [1,2]. These relations have also been shown to hold on surfaces of oxides, nitrides, sulfides, carbides, and functionalized graphitic materials [3-6]. Moreover, they provide the necessary atomic-scale insight to test rapidly reaction mechanisms, since they reduce the number of independent variables in microkinetic modeling, which ultimately allows determining trends in the catalytic activity of materials $[4,7-9]$. For the particular case of the electrochemical oxygen evolution or reduction reactions, these relations have been shown to impose a thermodynamic limitation on the performance of catalysts, which causes part of the large overpotential appearing during the operation of electrolyzers and fuel cells $[4,6,10]$. In spite of their fundamental importance and numerous applications, their origin is not clear and, therefore, the conditions under which they hold and the type of compounds to which they can be applied are not well understood. In this Letter, we take a step forward towards a deeper understanding of scaling relations that may lead to their further generalization. To this end, we have systematically studied the adsorption energies of second- and third-row atoms of groups 14-17 in the periodic table onto the near-surface alloys (NSAs) of $\mathrm{Pt}(111)$ and transition metals. The addition of guest atoms in the subsurface layers of a Pt host has been shown theoretically and confirmed experimentally to alter the electronic-structure properties of the Pt skin on the top layer [11-14], by this means modifying the catalytic performance of the host material.

All adsorption energies were calculated by means of density-functional theory (DFT) simulations using the VASP code [15] with the Perdew-Burke-Ernzerhof exchange-correlation functional [16]. The NSAs of $\operatorname{Pt}(111)$ and $3 d, 4 d$, and $5 d$ metals were represented by $2 \times 2$ supercells with a lattice constant of $3.98 \AA$. The Brillouin zones of all surfaces were sampled with $6 \times 6 \times 1$ Monkhorst-Pack grids [17]. Four metal layers were used, three of which contained only Pt atoms (the top and the two bottom layers) and one formed of transition metal atoms (the subsurface layer); the adsorbate coverage on the surface was, in all cases, 1/4 ML, and the adsorption site was atop (data for bridge and hollow sites are presented in Ref. [18]). The kinetic-energy cutoff for the plane-wave basis set was $450 \mathrm{eV}$. More calculation details can be found in Ref. [18]. The DFT adsorption energies of single atoms (A) were calculated relative to the clean surfaces and the isolated atoms, according to Eqs. (1) and (2):

$$
\begin{gathered}
*+A \rightarrow * A, \\
\Delta E_{A}=E_{* A}-E_{*}-E_{A} .
\end{gathered}
$$

Here $*$ denotes an active site in the surface, and $* A$ represents the adsorbed atom $A$. In the following, this atom may be $\mathrm{C}, \mathrm{Si}, \mathrm{N}, \mathrm{P}, \mathrm{O}, \mathrm{S}, \mathrm{F}$, or $\mathrm{Cl}$. The scaling relation between the energetics of a given pair of species 1 and 2 is expressed in mathematical terms as [1]

$$
\Delta E_{1}=\gamma \Delta E_{2}+\xi
$$

Given that $\gamma$ and $\xi$ are constant for a given set of adsorbates 1 and 2 for a given adsorption site on a given facet, $\Delta E_{1}$ depends explicitly only on $\Delta E_{2}$, and thus there is no direct dependence on any electronic-structure parameter in Eq. (3).

So far, scaling relations have mostly been shown to hold for adsorbed atoms and their hydrogenated species [3-6], and scalability between $* \mathrm{O}$ and $* \mathrm{Cl}$ has also been observed on (110) surfaces of rutile oxides [19]. Thus, it is of paramount importance to find out whether they exist between any other classes of adsorbed species and, if so, to determine the reason why that occurs. Figure 1 sheds light on the first question. In it, we present the scaling relations 


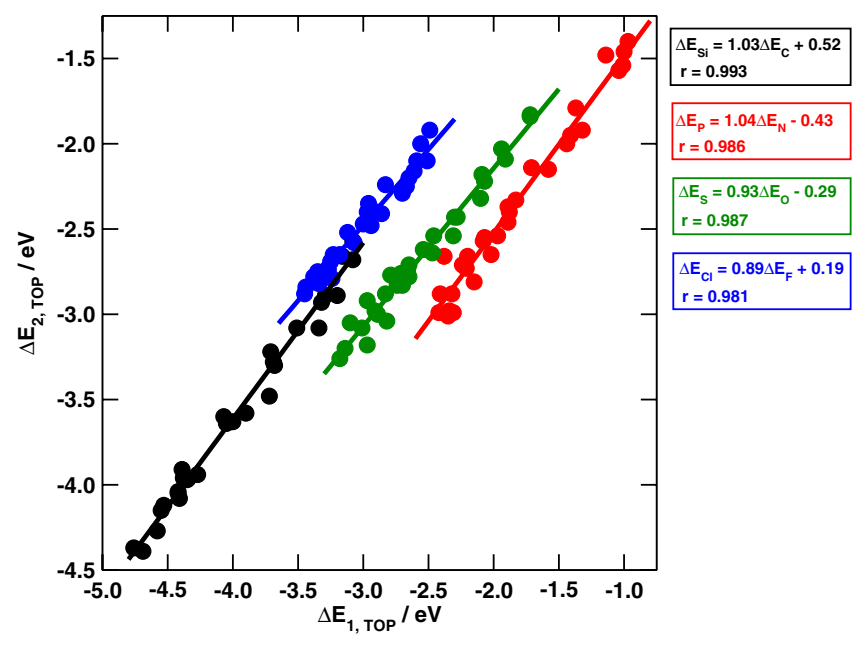

FIG. 1 (color online). Scaling relations between adsorption energies of single atoms atop NSAs of Pt and transition metals. In all cases the slopes are $\sim 1$, suggesting that each pair of adsorbates binds in the same way, so that the binding energies for a given surface differ only on a constant amount of energy. Data for bridge and hollow sites are provided in Ref. [18].

between the adsorption of second- and third-row atoms belonging to groups 14-17 of the periodic table on atop sites of NSAs of $\mathrm{Pt}(111)$ and $3 d, 4 d$, and $5 d$ metals (bridge and hollow data are shown in Ref. [18]). Although for chemical applications adsorption on bridge and hollow sites may be more relevant, the specific relationship that we wish to demonstrate here between scalability and electronic structure is clearest and "cleanest" for atop sites. Very similar scaling relations are found for bridge and hollow sites (see [18]) but with some additional features, the detailed discussion of which will be the subject of a future publication. We also emphasize that scaling relations are not restricted to species occupying the same adsorption sites, and thus scalability may exist between, e.g., an adsorbate on atop sites and another on hollows (see [18]).

Figure 1 shows that the adsorption energies of $\mathrm{C}^{*}$ and $\mathrm{Si}^{*}, \mathrm{~N}^{*}$ and $\mathrm{P}^{*}, \mathrm{O}^{*}$ and $\mathrm{S} *$, and $\mathrm{F}^{*}$ and $\mathrm{Cl}^{*}$ scale linearly and that the slope is in each case $\sim 1$, reflecting the fact that an identical coupling to the surface leads to a scaling relation between a given pair of adsorbates. For scaling relations between single atoms and their hydrogenated species [1], the slope was defined as $\gamma=\left(x_{\max }-x\right) / x$, where $x$ is the number of hydrogen atoms bonding to the considered adsorbed species and $x_{\max }$ is the maximum number of hydrogen atoms that can bond to the atom. However, the evidence provided in Fig. 1 confirms that scalability is possible for adsorbates that bind similarly to surfaces, regardless of the presence or absence of bonds to hydrogen atoms. Therefore, we propose that the slope of the scaling relations can be more generally defined as the ratio between the number of bonds that species 1 lacks to reach its valence number and the number of bonds that species 2 lacks to reach its valence number. Since this concept is more general, it covers, of course, the particular case of atoms and their hydrogenated species. Now, the reason for the existence of generalized scaling relations must stem from the correlation between adsorption energies and surface electronic-structure parameters, which is the basis of the surface-science approach to understanding heterogeneous processes at surfaces [2,11,20-22]. Thus, if the adsorption energies of species 1 and 2 depend upon a set of electronic-structure parameters, denoted as $\left\{\omega_{i}\right\}$, with $i=1,2,3, \ldots$, and the variations are described by functions $f$ and $g$, so that $\Delta E_{1}=f\left(\left\{\omega_{i}\right\}\right)+\alpha_{0}$ and $\Delta E_{2}=$ $g\left(\left\{\omega_{i}\right\}\right)+\beta_{0}$, the existence of a scaling relation in the way shown in Eq. (3) strictly requires that Eq. (4) be fulfilled:

$$
f\left(\left\{\omega_{i}\right\}\right)=\gamma g\left(\left\{\omega_{i}\right\}\right) .
$$

Equation (4) is essentially a reformulation of Eq. (3) which provides additional physicochemical insight, since it directly links electronic structure and energetic scalability. One of the consequences of Eq. (4) is that scaling relations hold for any set of adsorbates that satisfy its condition, as shown above in Fig. 1. Besides, if species 1 and 2 fulfill the condition in Eq. (4), we find that the offset in Eq. (3) depends on the slope $\gamma$ in the following way:

$$
\xi=\alpha_{0}-\gamma \beta_{0} .
$$

Further insight in the mathematical nature of the functions $f$ and $g$ can be provided by their critical points, i.e., maxima, minima, and saddle points. The simple process of taking the first derivative with respect to any parameter from the set $\left\{\omega_{i}\right\}$ and equaling the result to zero leads to the conclusion that $f$ and $g$ must as well share the same critical points for scalability to be possible.

We conclude that generalized scaling relations result from a smooth overlap between two curves that depend similarly on the same parameters. Nevertheless, we have not yet discussed which electronic-structure parameters of the set $\left\{\omega_{i}\right\}$ would be the most suitable ones to study the functions $f$ and $g$. In order to do so, it is worth noting that, when an atom is adsorbed on a series of different NSAs, the only change comes from the second layer, where the guest transition metal is located. Thus, the simplest electronicstructure parameters one can think of to describe the variations in adsorption energies are the group numbers (or the valence electrons) of $\mathrm{Pt}$, the guest element in the second layer, and the adsorbate. Figure 2 confirms that the sum of these parameters, which we will refer to as the total valence electrons, indeed captures smoothly the trends in adsorption energies of $\mathrm{C}, \mathrm{Si}, \mathrm{N}, \mathrm{P}, \mathrm{O}, \mathrm{S}, \mathrm{F}$, and $\mathrm{Cl}$ on NSAs with $3 d, 4 d$, and $5 d$ elements.

A closer look at Fig. 2 reveals that the minima of the adsorption energies are located in all cases at a total number of valence electrons of 24 (this is also the case for most adsorbates on bridge and hollow sites, though some exceptions exist for elements in groups 15 and 16). 

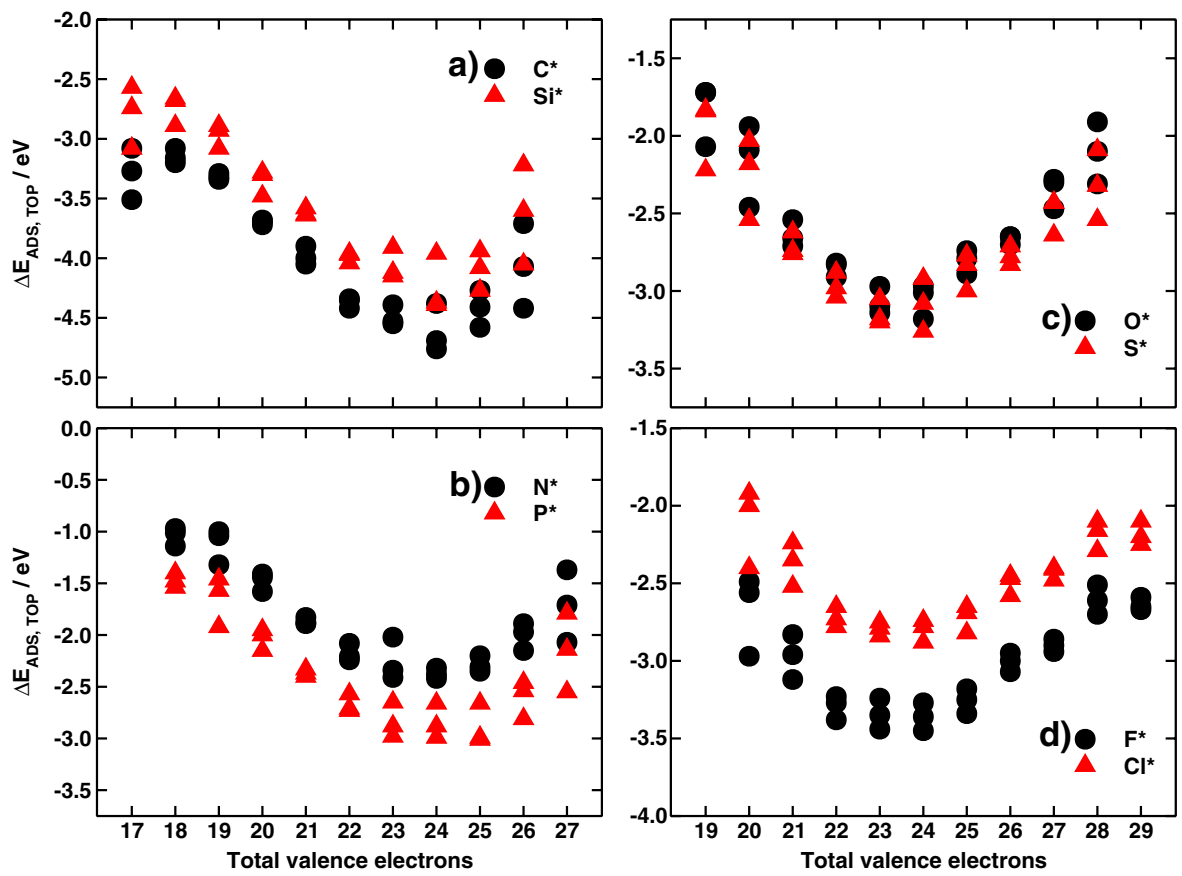

FIG. 2 (color online). Trends in adsorption energies of single atoms atop NSAs of Pt and transition metals: (a) $\mathrm{C}^{*}$ and $\mathrm{Si}^{*}$, (b) $\mathrm{N}^{*}$ and $\mathrm{P}^{*}$, (c) $\mathrm{O}^{*}$ and $\mathrm{S}^{*}$, and (d) $\mathrm{F}^{*}$ and $\mathrm{Cl}^{*}$. Circles denote $2 p$ elements $(\mathrm{C}, \mathrm{N}, \mathrm{O}$, and $\mathrm{F}$ ), and triangles denote $3 p$ elements $(\mathrm{Si}, \mathrm{P}, \mathrm{S}$, and $\mathrm{Cl}$ ). The total valence electrons are the sum of those of $\mathrm{Pt}$, the adsorbate, and the guest element in the second layer. The minima in all cases correspond to a total valence electron number of 24 in which the electronic configurations of both the adsorbates and the surface components resemble those of noble gases.

Since Pt has 10 valence electrons, $\mathrm{C}$ and $\mathrm{Si}$ have both 4 valence electrons, $\mathrm{N}$ and $\mathrm{P}$ have $5, \mathrm{O}$ and $\mathrm{S}$ have 6 , and $\mathrm{F}$ and $\mathrm{Cl}$ have 7 , we find that the minimum in adsorption energy among the NSAs is located in each case at a different guest metal, being the minimum at 10 for $\mathrm{C}^{*}$ and $\mathrm{Si}^{*}$ (corresponding to $\mathrm{Ni}, \mathrm{Pd}$, and $\mathrm{Pt}$ ), 9 for $\mathrm{N}^{*}$ and $\mathrm{P}^{*}$ (corresponding to $\mathrm{Co}, \mathrm{Rh}$, and Ir), 8 for $\mathrm{O}^{*}$ and $\mathrm{S}^{*}$ (corresponding to $\mathrm{Fe}, \mathrm{Ru}$, and $\mathrm{Os}$ ), and 7 for $\mathrm{F}^{*}$ and $\mathrm{Cl}^{*}$ (corresponding to $\mathrm{Mn}, \mathrm{Tc}$, and $\mathrm{Re}$ ). This constant number of electrons can be understood in terms of the 8- and 18-electron rules for the adsorbates and the surface components, respectively. These rules are well-known concepts in inorganic chemistry [23-25]. The idea underlying these rules is that when the electron cloud surrounding an atom resembles that of a noble gas, the stability is optimized [23-25]. For instance, consider the case of the minimum in adsorption energies of $\mathrm{C}^{*}$ in Fig. 2(a): C has 4 valence electrons while Pt has 10, so the number of valence electrons of the guest must be 10 , corresponding to $\mathrm{Ni}, \mathrm{Pd}$, or Pt. Assuming that the $\mathrm{C}$ adsorbate couples to $\mathrm{Pt}$ in the top layer in a fourfold way, which makes it achieve a noble gas configuration $(\mathrm{Ne})$ and follow the octet rule, that $\mathrm{Pt}$ couples to the guest, e.g., $\mathrm{Ni}$, in the second layer in a fourfold way, thus fulfilling the 18electron rule (resembling $\mathrm{Rn}$ ), and additionally that the $\mathrm{Ni}$ couples to the Pt atom in the third layer in a fourfold way, thereby fulfilling the 18-electron rule (resembling $\mathrm{Kr}$ ), we conclude that the system C-Pt-Ni has each component in its most stable electronic configuration. Lewis diagrams for all minima in Fig. 2 are provided in Fig. 3.

Figures 2 and 3 suggest that the adsorption energies appear to be straightforward functions of both the number of valence electrons of the components of the surface and that of the adsorbate. Moreover, the addition of both numbers is a fixed constant, which enables predictions in other systems like NSAs of $\mathrm{Au}$ and transition metals or pure metals. Therefore, these three parameters are key electronic-structure parameters of the set $\left\{\omega_{i}\right\}$ that governs the variations of the functions $f$ and $g$ in Eq. (4). Moreover, the fact that in each panel of Fig. 2 the vertical separation between the curves is constant implies that $E_{* 1}-E_{* 2}$, i.e., the energetic difference between the adsorbed states, is also approximately constant and has an average value of $0.76 \mathrm{eV}$. This means that the ways each pair of adsorbates in Fig. 2 couple to the surface are identical, and hence the difference in adsorption energies comes from the difference in orbital energies and sizes of species 1 and 2 (from $2 p$ to $3 p$ ). In Ref. [18], we also provide data for the adsorption of B and $\mathrm{Al}$ which agree with the trends shown here.

Finally, we address the problem of nonscalability between adsorbed species. Having established the rules shown above, it is possible to rectify the nonscalability in a simple way: If, for instance, one assumes that $f$ and $g$ are third-order functions of the total number of valence 


\begin{tabular}{ccccc}
\hline C & $\ddot{N}$ & $: 0:$ & $: \ddot{F}:$ & Adsorbates \\
|||| & || $\mid$ & || & $\mid$ & \\
$\mathbf{P t}:$ & $\mathbf{P t}:$ & $\mathbf{P t}$ & $\mathbf{P t}$ & $\mathbf{1}^{\text {st }}$ layer \\
|||| & |||| $\mid$ & ||||||$\downarrow$ & ||||||$\downarrow$ & \\
$\mathrm{Ni}:$ & $\mathbf{C o}$ & $\mathbf{F e}$ & $\mathbf{M n}$ & $2^{\text {nd }}$ layer \\
|||| & |||| & || & $\uparrow$ & \\
$\mathbf{P t}$ & $\mathbf{P t}$ & $\mathbf{P t}$ & $\mathbf{P t}$ & $\mathbf{3}^{\text {rd }}$ layer
\end{tabular}

FIG. 3 (color online). Lewis diagrams for the hypothetical systems $A$-Pt- $M$ ( $M$ being a $3 d$ metal) corresponding to the minima in Fig. 1. In all cases, the adsorbate follows the octet rule, and $\mathrm{Pt}$ and $\mathrm{M}$ follow the 18-electron rule, thus maximizing the stability of the system. Red lines are used to indicate shared pairs of electrons, while represents lone pairs, and arrows represent dative bonds.

electrons of the components of the NSAs, $\omega_{T}$, so that the adsorption energies are expressed as $\Delta E_{1}=\alpha_{0}+$ $\alpha_{1} \omega_{T_{1}}+\alpha_{2} \omega_{T_{1}}^{2}+\alpha_{3} \omega_{T_{1}}^{3}$ and $\Delta E_{2}=\beta_{0}+\beta_{1} \omega_{T_{2}}+$ $\beta_{2} \omega_{T_{2}}^{2}+\beta_{3} \omega_{T_{2}}^{3}$, then Eq. (3) turns into the following expression:

$$
\Delta E_{1}=\gamma \Delta E_{2}+\sum_{j=0}^{3}\left[\alpha_{j} \omega_{T_{1}}^{j}-\gamma \beta_{j} \omega_{T_{2}}^{j}\right]
$$

According to this equation, the scalability should be recovered provided that the third-order regression fits well the points. In order to test this statement, we consider the adsorption energies of $\mathrm{N}^{*}$ and $\mathrm{O}^{*}$. Based on Fig. 2, they should not scale, given that their curves have different shapes and their energetic minima are located at transition metals with different electron numbers. This is what is shown in Fig. 4, where we have put labels to the points to facilitate the detection of the trends: The maximum and minimum energies in the $\hat{\mathbf{x}}$ axis correspond to the maximum and minimum energies in the plot of $\Delta E_{N}$ vs valence electrons (Pt-Y and Pt-Ir, respectively). Analogously, the maximum and minimum energies in the $\hat{\mathbf{y}}$ axis correspond to those of the plot of $\Delta E_{O}$ vs valence electrons (Pt-Y and Pt-Os, respectively). Thus, the two branches in Fig. 4 correspond to elements to the left and to the right of the minima in their respective adsorption energy vs valence electrons plots. A direct consequence of this is that two sets of adsorption energies need to have their minima at the same group of the periodic table for their scaling to be smooth.

On the other hand, in the inset in Fig. 4 we observe that the agreement between DFT-calculated adsorption energies and the results from Eq. (6) is evident, when a value of $2 / 3$ is used for $\gamma$ (given that $\mathrm{O} *$ makes a double bond to the surface and $\mathrm{N}^{*}$ makes 3 ). Moreover, the mean absolute error (MAE) between the DFT-calculated adsorption energies and those estimated by means of Eq. (6) is only $0.07 \mathrm{eV}$. In principle, Eq. (6) could be expressed in terms of



FIG. 4. O* adsorption energies vs those of $\mathrm{N}^{*}$ for NSAs of Pt with transition metals. Clearly, there is no linear scalability between the energetics of the two species. Inset: Comparison between the DFT-calculated $\mathrm{O} *$ adsorption energies ( $\hat{\mathbf{y}}$ axis) and the predictions from Eq. (6) ( $\hat{\mathbf{x}}$ axis). The MAE is only $0.07 \mathrm{eV}$.

any kind of functions, not only cubic polynomials. This correction to nonscalability between two adsorbed species could be used to reduce the number of parameters in bi- or tridimensional volcano plots (see, for instance, Ref. [7]) and, furthermore, to make these plots based on the number of valence electrons of both the surface and the reaction intermediates adsorbed on the surface. It is noteworthy that the simple electron-counting analysis shown here has also proved successful in capturing the energetic trends in such diverse systems as functionalized graphitic materials and oxides, illustrating the role of periodicity in the properties of materials $[6,26,27]$. Nevertheless, it is worth noting that one of the reasons why we observe smooth trends in the plots of binding energies vs valence electrons is due to configurational correlations, since all surfaces with and without adsorbates are geometrically similar [28]. Moreover, we do not exclude that some other parameters such as band moments, characteristic $d$ radii, Pauli repulsion, or matrix elements may be part of the set of key parameters that determine the variations of adsorption properties throughout the $d$ series [11,29]. More information on this respect can be found in Ref. [18], where we present projected densities of states, $d$-band centers and fillings, and relevant geometric data for the NSAs with $3 d$ metals. In conclusion, we have shown here the existence of generalized scaling relations that apply to any species that bind similarly to surfaces. We found that the underlying physics and chemistry behind scaling relations are closely related to the overall electronic stability of the systems, and a simple solution to rectify nonscalability was proposed and tested with remarkable outcomes.

F. C.-V. and M. T. M. K. acknowledge financial support from the Netherlands Organization for Scientific Research 
(NWO) and the National Research School Catalysis (NRSC). J.I.M. acknowledges funding from Spanish MICINN through Juan de la Cierva Program.

*m.koper@chem.leidenuniv.nl

[1] F. Abild-Pedersen et al., Phys. Rev. Lett. 99, 016105 (2007).

[2] J. K. Nørskov et al., Nature Chem. 1, 37 (2009).

[3] E. M. Fernández et al., Angew. Chem., Int. Ed. 47, 4683 (2008).

[4] I. C. Man et al., ChemCatChem 3, 1159 (2011).

[5] A. Vojvodic et al., Phys. Rev. Lett. 103, 146103 (2009).

[6] F. Calle-Vallejo, J. I. Martínez, and J. Rossmeisl, Phys. Chem. Chem. Phys. 13, 15639 (2011).

[7] L. C. Grabow et al., Angew. Chem., Int. Ed. 50, 4601 (2011).

[8] G. Jones et al., Chem. Eng. Sci. 66, 6318 (2011).

[9] F. Studt et al., Science 320, 1320 (2008).

[10] M. T. M. Koper, J. Electroanal. Chem. 660, 254 (2011).

[11] J. R. Kitchin et al., J. Chem. Phys. 120, 10240 (2004).

[12] I.E. L. Stephens et al., J. Am. Chem. Soc. 133, 5485 (2011).

[13] J. Knudsen et al., J. Am. Chem. Soc. 129, 6485 (2007).

[14] K. J. Andersson et al., J. Am. Chem. Soc. 131, 2404 (2009).
[15] G. Kresse and J. Furthmuller, Phys. Rev. B 54, 11169 (1996).

[16] J. P. Perdew, K. Burke, and M. Ernzerhof, Phys. Rev. Lett. 77, 3865 (1996).

[17] H. J. Monkhorst and J. D. Pack, Phys. Rev. B 13, 5188 (1976).

[18] See Supplemental Material at http://link.aps.org/ supplemental/10.1103/PhysRevLett.108.116103 for the full calculation details, data for adsorption on bridge and hollow sites, a geometrical analysis of the trends shown in the Letter, projected densities of states and band centers for the NSAs, and data for the adsorption of B and Al.

[19] H. A. Hansen et al., Phys. Chem. Chem. Phys. 12, 283 (2010).

[20] B. Hammer and J. K. Nørskov, Adv. Catal. 45, 71 (2000).

[21] J. R. Kitchin et al., Phys. Rev. Lett. 93, 156801 (2004).

[22] R. A. van Santen, M. Neurock, and S. G. Shetty, Chem. Rev. 110, 2005 (2010).

[23] C. A. Tolman, Chem. Soc. Rev. 1, 337 (1972).

[24] P. R. Mitchell and R. V. Parish, J. Chem. Educ. 46, 811 (1969).

[25] D.F. Shriver and P.W. Atkins, Inorganic Chemistry (Oxford University, Oxford, 1999), 3rd ed.

[26] D. J. Mowbray et al., J. Phys. Chem. C 115, 2244 (2011).

[27] F. Calle-Vallejo et al., Angew. Chem., Int. Ed. 49, 7699 (2010).

[28] S. D. Miller et al., J. Chem. Phys. 134, 104709 (2011).

[29] H. Xin and S. Linic, J. Chem. Phys. 132, 221101 (2010). 